\title{
Actuator saturation and control design for buildings structural systems with improved uncertainty description
}

\author{
Y.C. Ding ${ }^{\mathrm{a}}$, F.L. Weng ${ }^{\mathrm{b}, *}$ and Z.A. Yu ${ }^{\mathrm{b}}$ \\ ${ }^{a}$ School of Resources and Environmental Engineering, Jiangxi University of Science and Technology, Ganzhou, \\ Jiangxi, China \\ ${ }^{\mathrm{b}}$ Faculty of Electrical Engineering and Automation, Jiangxi University of Science and Technology, Ganzhou, \\ Jiangxi, China
}

Received 27 August 2011

Revised 29 August 2012

Accepted 30 August 2012

\begin{abstract}
The problem of robustly active vibration control for a class of earthquake-excited structural systems with time-delay and saturation in the control input channel and parameter uncertainties appearing in all the mass, damping and stiffness matrices is concerned in this paper. The objective of the designing controllers is to guarantee the robust stability of the closed-loop system and attenuate the disturbance from earthquake excitation. Firstly, by using the linear combination of some matrices to deal with the system's uncertainties, a new system uncertainties description, namely rank-1 uncertainty description, is presented. Then, by introducing a linear varying parameter, the input saturation model is described as a linear parameter varying model. Furthermore, based on parameter-dependent Lyapunov theory and linear matrix inequality (LMI) technique, the LMIs-based conditions for the closed-loop system to be stable are deduced. By solving those conditions, the controller, considering the actuator saturation, input delay and parameters uncertainties, is obtained. Finally, a three-storey linear building structure under earthquake excitation is considered and simulation results are given to show the effectiveness of the proposed controllers.
\end{abstract}

Keywords: Buildings structure, actuator saturation, rank-1 uncertainty description, time-delay, earthquake

\section{Introduction}

As buildings become higher and higher, structural stability and solidity are challenged and cannot be guaranteed only by architectural materials of high quality. Therefore, the status of active control for seismic-excited or windexcited buildings becomes more and more significant [1]. Over the last few decades, great strides have been made in advancing the theory and practice of active structural control technology, such as classical $H_{\infty}$ theories [2,3], sliding mode control [4], neural networks [5], passive control [6], optimal control [7], Finite frequency $H_{\infty}$ control [8], etc., having been developed to attenuate building vibration under earthquake or wind excitation. In the meantime, some theoretical achievements have been transferred into practical applications, for example, active brace system (ABS) [9,10], and active mass damper (AMD) [11] have been widely studied and used for vibration attenuation.

${ }^{*}$ Corresponding author: F.L. Weng, Faculty of Electrical Engineering and Automation, Jiangxi University of Science and Technology, Ganzhou 341000, Jiangxi, China. Tel.: +86 797831 2506; Fax: +86 797831 2506; E-mail: wengfalu@ hotmail.com. 


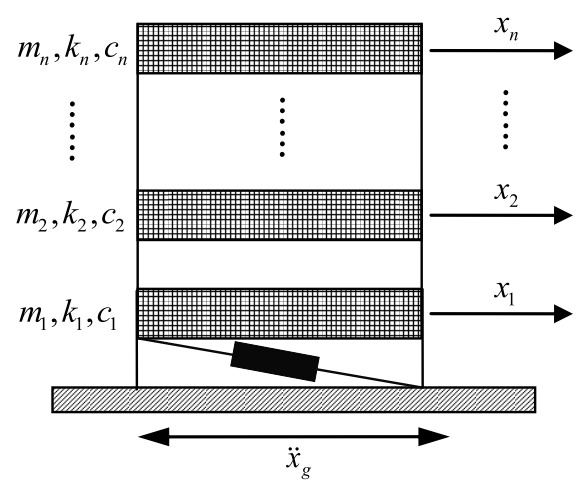

Fig. 1. $n$-story building model with ABS.

In particular, unavoidable input time delay often appears in buildings structural control systems. The time delay can be caused by data acquisition from sensors, filtering, processing of data, calculating control forces and transmitting the control force signals from computer to the actuator, actuator response, A/D, and D/A conversions, etc. [12]. Though the delay time may be short, it can nevertheless limit the control performance or even cause the instability of the system when the delay appears in the control channel [13,14]. Furthermore, most of the actuation devices are subject to amplitude saturation, that for the physical inputs such as force, torque, thrust, stroke, voltage, current, and flow rate of all conceivable applications of current technology are ultimately limited, and unexpected large amplitude disturbances can also push a system's actuators into saturation, thus forcing the system to operate in a nonlinear mode, for which it was not designed and in which it may be unstable [15]. Thus controller design for buildings structural systems, which involves actuator saturation, is also needed. Additionally, uncertainties, such as the inaccuracies of model parameters and the errors of sensors and actuators etc., almost exist in all practical systems; and the uncertain system with the stabilizing controller, which does not consider the parameter uncertainties, may lose stability $[16,17]$.

In this paper, based on utilizing the linear combination of some matrices, a new type of uncertainties' description, namely rank-1 uncertainty description, is presented. Furthermore, a linear varying parameter is employed to deal with the input saturation, and the system is transformed into a linear parameter varying (LPV) model. Based on parameter-dependent Lyapunov theory and linear matrix inequality (LMI) technique, the proposed sufficient conditions for the system to be stabilizable are expressed in a set of coupled LMIs. By solving these LMIs, the state-feedback controller, which is tolerant toward the actuator saturation, input time delay and parameter uncertainty while reduces the vibration of structural systems for external disturbance, can be easily obtained. In the end, simulation results are given to show the effectiveness of the proposed controllers.

The organization of this paper is as follows. Section 2 formulates the problem and presents the dynamic models. The main results are given in Section 3. The illustrative examples are expressed in Section 4 to show the applicability of the presented approaches. Finally, the paper is concluded in Section 5.

Notation: Throughout this paper, for real matrices $X$ and $Y$, the notation $X \geqslant Y$ (respectively, $X>Y$ ) means that the matrix $X-Y$ is semi-positive definite (respectively, positive definite). $I$ is the identity matrix with appropriate dimension, and a superscript " $T$ " represents transpose. For a symmetric matrix, $*$ denotes the symmetric terms. The symbol $R^{n}$ stands for the $n$-dimensional Euclidean space, and $R^{n \times m}$ is the set of $n \times m$ real matrices. $\|\omega\|_{2}$ expresses the 2-norm of $\omega$. We define $(M)^{H} \triangleq M+M^{T}$. A nonzero matrix $W$ is said to be rank-1 if there exist vectors $\xi_{1}$ and $\xi_{2}$ such that $W=\xi_{1} \xi_{2}^{T}$.

\section{Problem formulation and dynamic models}

Consider an $n$ degree-of-freedom linear building structure with an active brace system (ABS) installed in the first-storey unit, as shown in Fig. 1. 
The equation of motion, subjected to horizontal earthquake excitation, can be written as $[2,3,13,18]$

$$
\bar{M} \ddot{X}(t)+\bar{C} \dot{X}(t)+\bar{K} X(t)=\bar{H} u(t)+\bar{D} \ddot{x}_{g}(t),
$$

where $X(t)=\left[x_{1}(t), x_{2}(t), \cdots, x_{n}(t)\right]^{T}, x_{n}(t)$ is the relative displacement of the $n$th floor to ground; $u(t)$ is the control forces, and $\ddot{x}_{g}(t)$ is the earthquake ground acceleration; $\bar{M}, \bar{C}, \bar{K} \in R^{n \times n}$ are the mass, damping and stiffness matrices of the building, respectively. $\bar{H} \in R^{n \times 1}$ and $\bar{D} \in R^{n \times 1}$ are the controller location matrix and external excitation vector, respectively. From Fig. 1, we can obtain

$$
\begin{aligned}
& \bar{M}=\operatorname{diag}\left\{m_{1}, m_{2}, \cdots m_{n}\right\}, \quad \bar{H}=[1, \underbrace{0, \cdots 0}_{n-1}]^{T}, \quad \bar{D}=\left[-m_{1}-m_{2} \cdots-m_{n}\right]^{T}, \\
& \bar{C}=-\left[\begin{array}{ccccc}
-c_{1}-c_{2} & c_{2} & 0 & 0 \cdots 0 & 0 \\
c_{2} & -c_{2}-c_{3} & c_{3} & 0 \cdots 0 & 0 \\
\cdots & \cdots & & \cdots & \\
0 & \cdots & 0 & c_{n} & -c_{n}
\end{array}\right], \quad \bar{K}=-\left[\begin{array}{ccccc}
-k_{1}-k_{2} & k_{2} & 0 & 0 \cdots 0 & 0 \\
k_{2} & -k_{2}-k_{3} & k_{3} & 0 \cdots 0 & 0 \\
\cdots & \cdots & & \cdots & \\
0 & \cdots & 0 & k_{n} & -k_{n}
\end{array}\right] .
\end{aligned}
$$

In the state-space representation, the system Eq. (1) can be expressed as

$$
E \dot{x}(t)=A x(t)+B u(t)+B_{\omega} \omega(t),
$$

where

$$
x(t)=\left[\begin{array}{c}
X(t) \\
\dot{X}(t)
\end{array}\right], E=\left[\begin{array}{cc}
I & 0 \\
0 & \bar{M}
\end{array}\right], A=\left[\begin{array}{cc}
0 & I \\
-\bar{K} & -\bar{C}
\end{array}\right], B=\left[\begin{array}{c}
0 \\
\bar{H}
\end{array}\right], B_{\omega}=\left[\begin{array}{c}
0 \\
\bar{D}
\end{array}\right], \omega(t)=\ddot{x}_{g}(t) .
$$

In practice, the mass, damping and stiffness are usually subjected to possible perturbations, such as measurement error, the changes in environmental temperature and plastic deformation, etc. By assuming that the uncertain $m_{j} \in$ $\left[\underline{m}_{j}, \bar{m}_{j}\right], k_{j} \in\left[\underline{k}_{j}, \bar{k}_{j}\right]$, and $c_{j} \in\left[\underline{c}_{j}, \bar{c}_{j}\right]$, and denoting

$$
\begin{aligned}
& \hat{m}_{j}=\frac{1}{2}\left(\underline{m}_{j}+\bar{m}_{j}\right), \Delta \hat{m}_{j}=\theta_{1 j} \hat{m}_{j},\left|\theta_{1 j}\right| \leqslant \bar{\theta}_{1 j}<1, \bar{\theta}_{1 j}=\left(\bar{m}_{j}-\underline{m}_{j}\right) /\left(\bar{m}_{j}+\underline{m}_{j}\right), j=1,2, \cdots n, \\
& \hat{k}_{j}=\frac{1}{2}\left(\underline{k}_{j}+\bar{k}_{j}\right), \Delta \hat{k}_{j}=\theta_{2 j} \hat{k}_{j},\left|\theta_{2 j}\right| \leqslant \bar{\theta}_{2 j}<1, \bar{\theta}_{2 j}=\left(\bar{k}_{j}-k_{j}\right) /\left(\bar{k}_{j}+\underline{k}_{j}\right), j=1,2, \cdots n, \\
& \hat{c}_{j}=\frac{1}{2}\left(\underline{c}_{j}+\bar{c}_{j}\right), \Delta \hat{c}_{j}=\theta_{2(n+j)} \hat{c}_{j},\left|\theta_{2(n+j)}\right| \leqslant \bar{\theta}_{2(n+j)}<1, \bar{\theta}_{2(n+j)}=\left(\bar{c}_{j}-\underline{c}_{j}\right) /\left(\bar{c}_{j}+c_{j}\right), j=1,2, \cdots n,
\end{aligned}
$$

we can describe the uncertain system with input time-delay and actuator saturation by state space equation of the form:

$$
\begin{aligned}
E\left(\theta_{1}\right) \dot{x}(t) & =A\left(\theta_{2}\right) x(t)+B u(t-\tau)+B_{\omega}\left(\theta_{1}\right) \omega(t), t>0, \\
z(t) & =C_{z} x(t), \\
x(t) & =\Phi(t), t \in[-\tau, 0] \\
u(t) & =\sigma(K x(t)),
\end{aligned}
$$

where $\Phi(t)$ is the initial condition on the segment $[-\tau, 0], \tau>0$ is the input time delay; $C_{z} \in R^{l \times 2 n}$ is a real constant matrix with appropriate dimensions; $E\left(\theta_{1}\right), A\left(\theta_{2}\right), B_{\omega}\left(\theta_{1}\right)$ are uncertain real matrices with appropriate dimensions satisfying

$$
E\left(\theta_{1}\right)=E_{0}+\sum_{j=1}^{n} \theta_{1 j} E_{j}, A\left(\theta_{2}\right)=A_{0}+\sum_{j=1}^{2 n} \theta_{2 j} A_{j}, B_{\omega}\left(\theta_{1}\right)=B_{\omega 0}+\sum_{j=1}^{n} \theta_{1 j} B_{\omega j},
$$


where

$$
\begin{aligned}
& E_{0}=\operatorname{diag}\{\underbrace{1,1, \cdots 1}_{n}, \hat{m}_{1}, \hat{m}_{2}, \cdots \hat{m}_{n}\}, E_{j}=\operatorname{diag}\{\underbrace{0, \cdots 0}_{n+j-1}, \hat{m}_{j}, \underbrace{0, \cdots 0}_{n-j}\}=\hat{m}_{j} e_{j} f_{j}^{T},(j=1,2, \cdots n), \\
& B_{\omega 0}=[\underbrace{0,0, \cdots, 0}_{n},-\hat{m}_{1},-\hat{m}_{2}, \cdots,-\hat{m}_{n}]^{T}, B_{\omega j}=[\underbrace{0, \cdots 0}_{n+j-1},-\hat{m}_{j}, \underbrace{0, \cdots 0}_{n-j}]^{T}=-\hat{m}_{j} p_{j} q_{j}^{T},(j=1,2, \cdots n),
\end{aligned}
$$

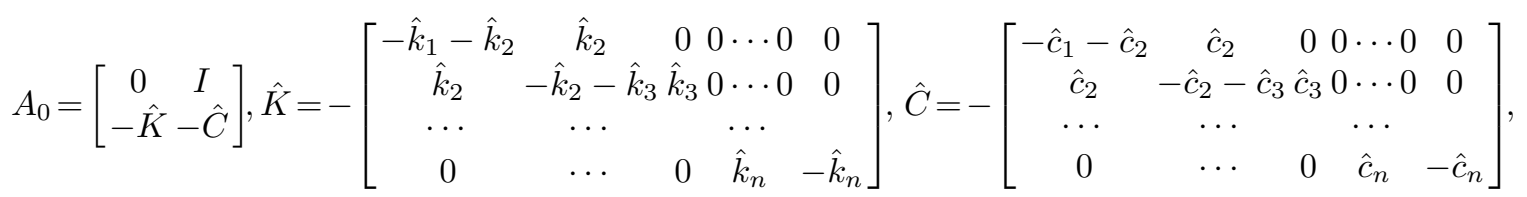

$$
\begin{aligned}
& A_{j}=\hat{k}_{j} a_{j} b_{j}^{T},(j=1,2, \cdots n), A_{n+j}=\hat{c}_{j} a_{n+j} b_{n+j}^{T},(j=1,2, \cdots, n),
\end{aligned}
$$

and $a_{j}, b_{j}, e_{j}, f_{j}, p_{j}, q_{j}$ are all column vectors with appropriate dimensions. $K \in R^{2 n}$ is the memoryless statefeedback controller gain that has to be designed. The saturation actuator is described by the following form introducing $\alpha(x(t))$ :

$$
u(t)=\sigma(K x(t))=\alpha(x(t)) \cdot K x(t)
$$

where $\alpha(x(t))=\frac{\sigma(K x(t))}{K x(t)}$, obviously, $0<\alpha(x(t)) \leqslant 1$.

Lemma 1: let $\mu, \nu$ and $F$ be real matrices of suitable dimensions with $F^{T} F \leqslant I$. Then, for any scalar $\sigma>0$,

$$
\mu F \nu+\nu^{T} F^{T} \mu^{T} \leqslant \sigma \mu \mu^{T}+\sigma^{-1} \nu^{T} \nu
$$

\section{Main results}

Based on the above ideas, the following theorems give the existence conditions of a stabilizing state-feedback controller for system Eq. (3) with the performance $\|z\|_{2}<\gamma\|\omega\|_{2}$.

Theorem 1: The system Eq. (3) without uncertainties is asymptotically stabilizable with the performance $\|z\|_{2}<$ $\gamma\|\omega\|_{2}$ for all non-zero $\omega \in L_{2}[0, \infty)$, constant time-delay $\tau$, if there exist positive definite matrices $P_{1}, P_{2}, R, Q_{1}$, $Q_{2}$ matrices $U, V, W, S, Z$ and scalars $\beta_{1}, \beta_{2}$ satisfying the following LMIs

$$
\Psi_{i}=\left[\begin{array}{ccccccc}
\psi_{i 11} & \psi_{i 12} & \psi_{i 13} & \tau P_{2}^{T} & \psi_{i 15} & B_{\omega 0} & S C_{z}^{T} \\
* & \psi_{22} & \psi_{23} & -\tau P_{2}^{T} & \psi_{i 25} & \beta_{2} B_{\omega 0} & 0 \\
* & * & \psi_{33} & 0 & \psi_{i 35} & \beta_{1} B_{\omega 0} & 0 \\
* & * & * & -\tau Q_{2} & 0 & 0 & 0 \\
* & * & * & * & -\tau Q_{1} & 0 & 0 \\
* & * & * & * & * & -\gamma^{2} I & 0 \\
* & * & * & * & * & * & -I
\end{array}\right]<0, \quad i=1,2
$$

where

$$
\begin{aligned}
& \psi_{111}=R+\tau Q_{2}+U+U^{T}+A_{0} S^{T}+B Z+S A_{0}^{T}+Z^{T} B^{T}, \psi_{112}=-U+W^{T}+\beta_{2} S A_{0}^{T}+\beta_{2} Z^{T} B^{T} \\
& \psi_{113}=\bar{P}_{1}+\bar{V}^{T}-E_{0} S^{T}+\beta_{1} S A_{0}^{T}+\beta_{1} Z^{T} B^{T}, \psi_{115}=-\tau U-\tau B Z, \psi_{22}=-R-W-W^{T} \\
& \psi_{23}=-V^{T}-\beta_{2} E_{0} S^{T}, \psi_{125}=-\tau W-\beta_{2} \tau B Z, \psi_{33}=\tau Q_{1}-\beta_{1} E_{0} S^{T}-\beta_{1} S E_{0}^{T}, \psi_{135}=-\tau V-\beta_{1} \tau B Z
\end{aligned}
$$




$$
\begin{aligned}
& \psi_{211}=R+\tau Q_{2}+U+U^{T}+A_{0} S^{T}+S A_{0}^{T}, \psi_{212}=-U+W^{T}+\beta_{2} S A_{0}^{T}, \\
& \psi_{213}=\bar{P}_{1}+\bar{V}^{T}-E_{0} S^{T}+\beta_{1} S A_{0}^{T}, \psi_{215}=-\tau U, \psi_{225}=-\tau W, \psi_{235}=-\tau V .
\end{aligned}
$$

Furthermore, one asymptotically stabilizing gain $K$ is chosen as

$$
K=Z S^{-T} .
$$

Proof: Multiply the above $i=1,2$ inequalities by $\alpha(x(t-\tau))$ and $(1-\alpha(x(t-\tau)))$ respectively, and sum to get

$$
\Psi=\left[\begin{array}{ccccccc}
\psi_{11} & \psi_{12} & \psi_{13} & \tau P_{2}^{T} & \psi_{15} & B_{\omega 0} & S C_{z}^{T} \\
* & \psi_{22} & \psi_{23} & -\tau P_{2}^{T} & \psi_{25} & \beta_{2} B_{\omega 0} & 0 \\
* & * & \psi_{33} & 0 & \psi_{35} & \beta_{1} B_{\omega 0} & 0 \\
* & * & * & -\tau Q_{2} & 0 & 0 & 0 \\
* & * & * & * & -\tau Q_{1} & 0 & 0 \\
* & * & * & * & * & -\gamma^{2} I & 0 \\
* & * & * & * & * & * & -I
\end{array}\right]<0,
$$

where

$$
\begin{aligned}
& \psi_{11}=R+\tau Q_{2}+U+U^{T}+A_{0} S^{T}+\alpha(x(t-\tau)) B Z+S A_{0}^{T}+\alpha(x(t-\tau)) Z^{T} B^{T}, \\
& \psi_{12}=-U+W^{T}+\beta_{2} S A_{0}^{T}+\alpha(x(t-\tau)) \beta_{2} Z^{T} B^{T}, \psi_{15}=-\tau U-\alpha(x(t-\tau)) \tau B Z, \\
& \psi_{13}=\bar{P}_{1}+\bar{V}^{T}-E_{0} S^{T}+\beta_{1} S A_{0}^{T}+\alpha(x(t-\tau)) \beta_{1} Z^{T} B^{T}, \psi_{25}=-\tau W-\beta_{2} \alpha(x(t-\tau)) \tau B Z, \\
& \psi_{35}=-\tau V-\beta_{1} \alpha(x(t-\tau)) \tau B Z .
\end{aligned}
$$

Define a parameter-dependent Lyapunov-Krasovskii functional candidate as

$$
\begin{aligned}
V(t)= & x(t)^{T} \bar{P}_{1} x(t)+\int_{t-\tau}^{t} x^{T}(\varepsilon) d \varepsilon \bar{P}_{2} \int_{t-\tau}^{t} x(\varepsilon) d \varepsilon+\int_{t-\tau}^{t} x^{T}(\sigma) \bar{R} x(\sigma) d s \\
& +\int_{-\tau}^{0} \int_{t+\varepsilon}^{t} \dot{x}^{T}(\sigma) \bar{Q}_{1} \dot{x}(\sigma) d s d \varepsilon+\int_{-\tau}^{0} \int_{t+\varepsilon}^{t} x^{T}(\sigma) \bar{Q}_{2} x(\sigma) d s d \varepsilon,
\end{aligned}
$$

where $\bar{P}_{i}>0(i=1,2), \bar{R}>0, \bar{Q}_{i}>0(i=1,2)$ are matrices to be determined. Then, the derivative of $V(t)$ along the solution of system Eq. (3) is given by

$$
\begin{aligned}
\dot{V}(t)= & 2 x(t)^{T} \bar{P}_{1} \dot{x}(t)+2 \int_{t-\tau}^{t} x^{T}(\varepsilon) d \varepsilon \bar{P}_{2}(x(t)-x(t-\tau)) \\
& +x^{T}(t) \bar{R} x(t)-x^{T}(t-\tau) \bar{R} x(t-\tau)+\tau \dot{x}^{T}(t) \bar{Q}_{1} \dot{x}(t) \\
& -\int_{t-\tau}^{t} \dot{x}^{T}(\varepsilon) \bar{Q}_{1} \dot{x}(\varepsilon) d \varepsilon+\tau x^{T}(t) \bar{Q}_{2} x(t)-\int_{t-\tau}^{t} x^{T}(\varepsilon) \bar{Q}_{2} x(\varepsilon) d \varepsilon
\end{aligned}
$$

For any matrices $\bar{U}, \bar{V}, \bar{W}$, we obtain

$$
2\left(x^{T}(t) \bar{U}+\dot{x}^{T}(t) \bar{V}+x^{T}(t-\tau) \bar{W}\right)\left(x(t)-x(t-\tau)-\int_{t-\tau}^{t} \dot{x}(\varepsilon) d \varepsilon\right)=0 .
$$

By assuming $\theta_{1 i}=0(i=1,2, \cdots n), \theta_{2 j}=0(j=1,2, \cdots 2 n)$, for any matrix $\bar{S}$, scalars $\beta_{1}, \beta_{2}$, we have

$$
\begin{array}{r}
\left(x^{T}(t) \bar{S}+\dot{x}^{T}(t) \beta_{1} \bar{S}+x^{T}(t-\tau) \beta_{2} \bar{S}\right)\left(A_{0} x(t)+\alpha(x(t-\tau)) B K x(t)\right. \\
\left.-E_{0} \dot{x}(t)-\alpha(x(t-\tau)) B K \int_{t-\tau}^{t} \dot{x}(\varepsilon) d \varepsilon+B_{\omega 0} \omega(t)\right)=0 .
\end{array}
$$


Next, we will establish the $\|z\|_{2}<\gamma\|\omega\|_{2}$ performance of the system Eq. (3) under zero initial condition, that is, $\Phi(t)=0, \forall t \in[-\tau, 0]$, and $\left.V(t)\right|_{t=0}=0$. Consider the following index:

$$
J \triangleq \int_{0}^{\infty}\left[Z^{T}(t) Z(t)-\gamma^{2} \omega(t)^{T} \omega(t)\right] d t .
$$

Then, for any non-zero $\omega \in L_{2}[0, \infty)$, there holds

$$
\begin{aligned}
J & \leqslant \int_{0}^{\infty}\left[Z^{T}(t) Z(t)-\gamma^{2} \omega(t)^{T} \omega(t)\right] d t+\left.V(t)\right|_{t=\infty}-\left.V(t)\right|_{t=0} \\
& =\int_{0}^{\infty}\left[Z^{T}(t) Z(t)-\gamma^{2} \omega(t)^{T} \omega(t)+\dot{V}(t)\right] d t
\end{aligned}
$$

Noting Eqs (11)-(15), after some algebraic manipulations, we obtain

$$
Z^{T}(t) Z(t)-\gamma^{2} \omega(t)^{T} \omega(t)+\dot{V}(t) \leqslant \frac{1}{\tau} \int_{t-\tau}^{t} \eta^{T}(t, \varepsilon) \Xi \eta(t, \varepsilon) d \varepsilon
$$

where $\eta(t, \varepsilon)=[x(t), x(t-\tau), \dot{x}(t), x(\varepsilon), \dot{x}(\varepsilon), \omega(t)]^{T}$ and

$$
\begin{aligned}
& \Xi=\left[\begin{array}{cccccc}
\Xi_{11} & \Xi_{12} & \Xi_{13} & \Xi_{14} & \Xi_{15} & \Xi_{16} \\
* & \Xi_{22} & \Xi_{23} & \Xi_{24} & \Xi_{25} & \Xi_{26} \\
* & * & \Xi_{33} & 0 & \Xi_{35} & \Xi_{36} \\
* & * & * & \Xi_{44} & 0 & 0 \\
* & * & * & * & \Xi_{55} & 0 \\
* & * & * & * & * & \Xi_{66}
\end{array}\right], \\
& \Xi_{11}=\bar{R}+\tau \bar{Q}_{2}+\bar{U}+\bar{U}^{T}+\bar{S}\left(A_{0}+\alpha(x(t-\tau)) B K\right)+\left(A_{0}+\alpha(x(t-\tau)) B K\right)^{T} \bar{S}^{T}+C_{z}^{T} C_{z}, \\
& \Xi_{12}=-\bar{U}+\bar{W}^{T}+\beta_{2}\left(A_{0}+\alpha(x(t-\tau)) B K\right)^{T} \bar{S}^{T}, \Xi_{13}=\bar{P}_{1}+\bar{V}^{T}-\bar{S} E_{0}+\beta_{1}\left(A_{0}+\alpha(x(t-\tau)) B K\right)^{T} \bar{S}^{T}, \\
& \Xi_{14}=\tau \bar{P}_{2}^{T}, \Xi_{15}=-\tau \bar{U}-\tau \alpha(x(t-\tau)) \bar{S} B K, \Xi_{16}=\bar{S} B_{\omega 0}, \Xi_{22}=-\bar{R}-\bar{W}-\bar{W}^{T}, \\
& \Xi_{23}=-\bar{V}^{T}-\beta_{2} \bar{S} E_{0}, \Xi_{24}=-\tau \bar{P}_{2}^{T}, \Xi_{25}=-\tau \bar{W}-\beta_{2} \alpha(x(t-\tau)) \tau \bar{S} B K, \Xi_{26}=\beta_{2} \bar{S} B_{\omega 0}, \\
& \Xi_{33}=\tau \bar{Q}_{1}-\beta_{1} \bar{S} E_{0}-\beta_{1} E_{0}^{T} \bar{S}^{T}, \Xi_{35}=-\tau \bar{V}-\beta_{1} \alpha(x(t-\tau)) \tau \bar{S} B K, \Xi_{36}=\beta_{1} \bar{S} B_{\omega 0}, \Xi_{44}=-\tau \bar{Q}_{2}, \\
& \Xi_{55}=-\tau \bar{Q}_{1}, \Xi_{66}=-\gamma^{2} I .
\end{aligned}
$$

Then, if $\Xi<0$, we have $J \leqslant \int_{0}^{\infty}\left[Z^{T}(t) Z(t)-\gamma^{2} \omega(t)^{T} \omega(t)+\dot{V}(t)\right] d t<0$, hence $\|z\|_{2}<\gamma\|\omega\|_{2}$ is satisfied. Assuming the zero-disturbance input, i.e. $\omega(t) \equiv 0$, if $\Xi<0$, we can easily obtain $\dot{V}(t)<0$, and the asymptotic stability of closed-loop system is established.

Pre- and post-multiplying $\Xi<0$ with $\operatorname{diag}\left(\bar{S}^{-1}, \bar{S}^{-1}, \bar{S}^{-1}, \bar{S}^{-1}, \bar{S}^{-1}, I\right)$ and its transpose respectively, and defining $P_{1}=\bar{S}^{-1} \bar{P}_{1} \bar{S}^{-T}, P_{2}=\bar{S}^{-1} \bar{P}_{2} \bar{S}^{-T}, Q_{1}=\bar{S}^{-1} \bar{Q}_{1} \bar{S}^{-T}, Q_{2}=\bar{S}^{-1} \bar{Q}_{2} \bar{S}^{-T}, R=\bar{S}^{-1} \bar{R} \bar{S}^{-T}, U=$ $\bar{S}^{-1} \bar{U} \bar{S}^{-T}, V=\bar{S}^{-1} \bar{V} \bar{S}^{-T}, W=\bar{S}^{-1} \bar{W} \bar{S}^{-T}, Z=K \bar{S}^{-T}$ and $S=\bar{S}^{-1}$, we can obtain $\Xi<0$ from Eq. (9) easily. This completes the proof.

Theorem 2: The uncertain system Eq. (3) is robustly stabilizable with the performance $\|z\|_{2}<\gamma\|\omega\|_{2}$ for all non-zero $\omega \in L_{2}[0, \infty)$, constant time-delay $\tau$, if there exist positive definite matrices $P_{1}, P_{2}, R, Q_{1}, Q_{2}$ matrices $U, V, W, S, Z$ and any positive scalars $v_{11}, v_{12}, \cdots, v_{1 n}, v_{21}, v_{22}, \cdots, v_{2(2 n)}, v_{31}, v_{32}, \cdots, v_{3 n}$ and scalars $\beta_{1}$, $\beta_{2}$, satisfying the following LMIs

$$
\left[\begin{array}{cc}
\Upsilon_{i} & \mathrm{M}_{12} \\
* & \mathrm{M}_{22}
\end{array}\right]<0, \quad(i=1,2)
$$


where $\Psi_{i}(i=1,2)$ follow the same definitions as those in Theorem 1 , and

$$
\begin{aligned}
& \Upsilon_{i}=\Psi_{i}+\sum_{j=1}^{n} v_{1 j} \hat{m}_{j}^{2} \bar{\theta}_{1 j}^{2} \Gamma_{1 j} \Gamma_{1 j}^{T}+\sum_{j=1}^{n} v_{2 j} \hat{k}_{j}^{2} \bar{\theta}_{2 j}^{2} \Gamma_{2 j} \Gamma_{2 j}^{T}+\sum_{j=1}^{n} v_{2(n+j)} \hat{c}_{j}^{2} \bar{\theta}_{2(n+j)}^{2} \Gamma_{2(n+j)} \Gamma_{2(n+j)}^{T} \\
& +\sum_{j=1}^{n} v_{3 j} \hat{m}_{j}^{2} \bar{\theta}_{1 j}^{2} \Gamma_{3 j} \Gamma_{3 j}^{T}
\end{aligned}
$$

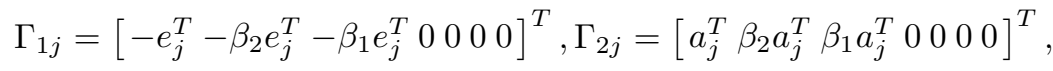

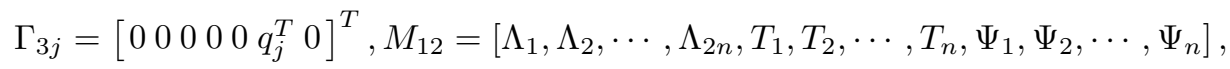

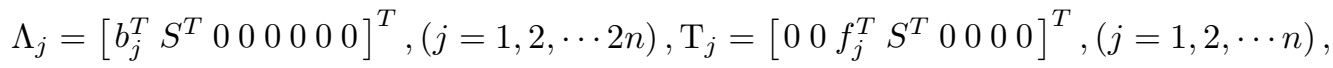

$$
\begin{aligned}
& \Psi_{j}=\left[\begin{array}{llllll}
p_{j}^{T} & \beta_{2} p_{j}^{T} \beta_{1} p_{j}^{T} & 0 & 0 & 0 & 0
\end{array}\right]^{T},(j=1,2, \cdots n), \\
& M_{22}=\operatorname{diag}\left\{-v_{21} I,-v_{22} I, \cdots-v_{2(2 n)} I,-v_{11} I,-v_{12} I, \cdots-v_{1 n} I,-v_{31} I,-v_{32} I, \cdots-v_{3 n} I\right\} .
\end{aligned}
$$

Furthermore, the state-feedback controller gain matrix description is the same as Eq. (8).

Proof: Replacing $E_{0}, A_{0}$ and $B_{\omega 0}$ with $E_{0}+\sum_{j=1}^{n} \theta_{1 j} E_{j}, A_{0}+\sum_{j=1}^{2 n} \theta_{2 j} A_{j}$, and $B_{\omega 0}+\sum_{j=1}^{n} \theta_{1 j} B_{\omega j}$ respectively, Eq. (7) can be expressed as

$$
\begin{aligned}
\Psi_{i} & +\sum_{j=1}^{n} \theta_{1 j}\left(\hat{m}_{j} \mathrm{~T}_{j} \Gamma_{1 j}^{T}\right)^{H}+\sum_{j=1}^{n} \theta_{1 j}\left(\hat{m}_{j} \Gamma_{3 j} \Psi_{j}^{T}\right)^{H}+\sum_{j=1}^{n} \theta_{2 j}\left(\hat{k}_{j} \Lambda_{j} \Gamma_{2 j}^{T}\right)^{H} \\
& +\sum_{j=1}^{n} \theta_{2(n+j)}\left(\hat{c}_{j} \Lambda_{(n+j)} \Gamma_{2(n+j)}^{T}\right)^{H}<0, \quad(i=1,2) .
\end{aligned}
$$

By Lemma 1, Eq. (18) holds if there exist positive scalars $v_{11}, v_{12}, \cdots, v_{1 n}, v_{21}, v_{22}, \cdots, v_{2(2 n)}, v_{31}, v_{32}, \cdots, v_{3 n}$ such that

$$
\begin{aligned}
\Psi_{i}+ & \sum_{j=1}^{n}\left(v_{1 j}^{-1} \mathrm{~T}_{j} \mathrm{~T}_{j}^{T}+v_{1 j} \bar{\theta}_{1 j}^{2} \hat{m}_{j}^{2} \Gamma_{1 j} \Gamma_{1 j}^{T}+v_{3 j}^{-1} \Psi_{j} \Psi_{j}^{T}+v_{3 j} \bar{\theta}_{1 j}^{2} \hat{m}_{j}^{2} \Gamma_{3 j} \Gamma_{3 j}^{T}+v_{2 j}^{-1} \Lambda_{j} \Lambda_{j}^{T}\right. \\
& \left.+v_{2 j} \bar{\theta}_{2 j}^{2} \hat{c}_{j}^{2} \Gamma_{2 j} \Gamma_{2 j}^{T}+v_{2(n+j)}^{-1} \Lambda_{n+j} \Lambda_{n+j}^{T}+v_{2(n+j)} \bar{\theta}_{2(n+j)}^{2} \hat{k}_{j}^{2} \Gamma_{2(n+j)} \Gamma_{2(n+j)}^{T}\right)<0,(i=1,2) .
\end{aligned}
$$

Applying the Schur complement, LMIs Eq. (19) is equivalent to LMIs Eq. (17). This completes the proof.

Furthermore, if there has no input time delay, the system Eq. (3) can be rewritten as

$$
\begin{aligned}
E\left(\theta_{1}\right) \dot{x}(t) & =A\left(\theta_{2}\right) x(t)+B u(t)+B_{\omega}\left(\theta_{1}\right) \ddot{x}_{g}, t>0, \\
z(t) & =C_{z} x(t) \\
u(t) & =\sigma(K x(t)) .
\end{aligned}
$$

The Lyapunov-Krasovskii functional candidate Eq. (10) is replaced by

$$
V(t)=x(t)^{T} P_{1} x(t)
$$

and Eq. (13) can be rewritten as

$$
2\left(x^{T}(t) \bar{S}+\dot{x}^{T}(t) \beta_{1} \bar{S}\right)\left(\left(A_{0}+B K\right) x(t)+B_{\omega 0} \omega(t)-E_{0} \dot{x}(t)\right)=0 .
$$


According to the proof of Theorems above, we obtain the following Corollaries.

Corollary 1: The system Eq. (20) without uncertainties is asymptotical stabilizable with the performance $\|z\|_{2}<$ $\gamma\|\omega\|_{2}$ for all non-zero $\omega \in L_{2}[0, \infty)$, if there exist $P>0, S, Z$, and a scalar $\beta$ satisfying the following LMIs

$$
\Pi_{i}=\left[\begin{array}{cccc}
\psi_{i 11} & \psi_{i 12} & B_{\omega 0} & S C_{z}^{T} \\
* & \psi_{22} & \beta B_{\omega 0} & 0 \\
* & * & -\gamma^{2} I & 0 \\
* & * & * & -I
\end{array}\right]<0, i=1,2,
$$

where

$$
\begin{aligned}
& \psi_{111}=A_{0} S^{T}+S A_{0}^{T}+B Z+Z^{T} B^{T}, \psi_{112}=P-E_{0} S^{T}+\beta S A_{0}^{T}+\beta Z^{T} B^{T}, \psi_{22}=-\beta S E_{0}^{T}-\beta E_{0} S^{T}, \\
& \psi_{211}=A_{0} S^{T}+S A_{0}^{T}, \psi_{212}=P-E_{0} S^{T}+\beta S A_{0}^{T} .
\end{aligned}
$$

Furthermore, the state-feedback controller gain matrix description is the same as Eq. (8).

Corollary 2: The system Eq. (20) is robustly stabilizable with the performance $\|z\|_{2}<\gamma\|\omega\|_{2}$ for all non-zero $\omega \in L_{2}[0, \infty)$, if there exist $P>0, S, Z$ positive scalars $v_{11}, v_{12}, \cdots, v_{1 n}, v_{21}, v_{22}, \cdots, v_{2(2 n)}, v_{31}, v_{32}, \cdots, v_{3 n}$ and a scalar $\beta$ satisfying the following LMIs

$$
\left[\begin{array}{cc}
\Theta_{i} & M_{12} \\
* & M_{22}
\end{array}\right]<0, \quad i=1,2
$$

where $\Pi_{i}(i=1,2)$ follow the same definitions as those in Corollary 1 , and

$$
\begin{aligned}
& \Theta_{i}=\Pi_{i}+\sum_{j=1}^{n} v_{1 j} \bar{\theta}_{1 j}^{2} \hat{m}_{j}^{2} \Gamma_{1 j} \Gamma_{1 j}^{T}+\sum_{j=1}^{n} v_{2 j} \bar{\theta}_{2 j}^{2} \hat{k}_{j}^{2} \Gamma_{2 j} \Gamma_{2 j}^{T} \\
& +\sum_{j=1}^{n} v_{2(n+j)} \bar{\theta}_{2(n+j)}^{2} \hat{c}_{j}^{2} \Gamma_{2(n+j)} \Gamma_{2(n+j)}^{T}+\sum_{j=1}^{n} v_{3 j} \bar{\theta}_{3 j}^{2} \hat{m}_{j}^{2} \Gamma_{3 j} \Gamma_{3 j}^{T}, \\
& \Gamma_{1 j}=\left[e_{j} \beta e_{j} 00\right]^{T}, \Gamma_{2 j}=\left[a_{j} \beta a_{j} 00\right]^{T}, \Gamma_{3 j}=\left[\begin{array}{lll}
0 & 0 & q_{j}
\end{array} 0\right]^{T}, \\
& M_{12}=\left[\begin{array}{cccccccccccc}
S b_{1} & S b_{2} & \cdots & S b_{2 n} & 0 & 0 & \cdots & 0 & p_{1} & p_{2} & \cdots & p_{n} \\
0 & 0 & \cdots & 0 & S f_{1} & S f_{2} & \cdots & S f_{n} & \beta p_{1} & \beta p_{2} & \cdots & \beta p_{n} \\
0 & 0 & \cdots & 0 & 0 & 0 & \cdots & 0 & 0 & 0 & \cdots & 0 \\
0 & 0 & \cdots & 0 & 0 & 0 & \cdots & 0 & 0 & 0 & \cdots & 0
\end{array}\right] \text {, } \\
& M_{22}=\operatorname{diag}\left\{-v_{21} I,-v_{22} I, \cdots-v_{2(2 n)} I,-v_{11} I,-v_{12} I, \cdots-v_{1 n} I,-v_{31} I,-v_{32} I, \cdots-v_{3 n} I\right\} .
\end{aligned}
$$

Furthermore, the state-feedback controller gain matrix description is the same as Eq. (8).

\section{Illustrative examples}

Example 1: A three-story scaled building model studied by $[10,19,20]$, in which every storey unit is identically constructed and an active brace system (ABS) is installed in the first-story unit, as shown in Fig. 1, is considered. The mass, stiffness and damping coefficient of each storey unit for nominal system are $m_{i}=1000 \mathrm{~kg}, k_{i}=980 \mathrm{kN} / \mathrm{m}$, and $c_{i}=1.407 \mathrm{kNs} / \mathrm{m}$, respectively, for $i=1,2,3$. This system can be described by state space Eq. (3), which has $x=\left[\begin{array}{llllll}x_{1} & x_{2} & x_{3} & \dot{x}_{1} & \dot{x}_{2} & \dot{x}_{3}\end{array}\right]^{T}$, control input vector $B=\left[\begin{array}{lllll}0 & 0 & 0 & 1 & 0\end{array}\right]^{T}$, control output vector $C_{z}=\left[\begin{array}{llllll}0 & 0 & 0 & 1 & 1 & 1\end{array}\right]$, and the system matrices $E\left(\theta_{1}\right), A\left(\theta_{2}\right)$ and $B_{\omega}\left(\theta_{1}\right)$ are as followings:

$$
E\left(\theta_{1}\right)=E_{0}+\theta_{11} E_{1}+\theta_{12} E_{2}+\theta_{13} E_{3}, B_{\omega}\left(\theta_{1}\right)=B_{\omega 0}+\theta_{11} B_{\omega 1}+\theta_{12} B_{\omega 2}+\theta_{13} B_{\omega 3},
$$


Table 1

Maximum response values for deterministic system

\begin{tabular}{|c|c|c|c|c|c|c|c|c|c|c|c|c|}
\hline \multirow[t]{2}{*}{ Storey } & \multicolumn{2}{|c|}{ No control } & \multicolumn{2}{|c|}{ LQR } & \multicolumn{2}{|c|}{$\mathrm{MBBC}$} & \multicolumn{2}{|c|}{ SSMC } & \multicolumn{2}{|c|}{ RSC } & \multicolumn{2}{|c|}{ Controller I } \\
\hline & $d_{i}$ & $\ddot{x}_{i}$ & $d_{i}$ & $\ddot{x}_{i}$ & $d_{i}$ & $\ddot{x}_{i}$ & $d_{i}$ & $\ddot{x}_{i}$ & $d_{i}$ & $\ddot{x}_{i}$ & $d_{i}$ & $\ddot{x}_{i}$ \\
\hline 1 & 1.34 & 3.13 & 0.88 & 2.25 & 0.51 & 1.64 & 0.52 & 1.62 & 0.53 & 1.60 & 0.53 & 1.56 \\
\hline 2 & 1.02 & 4.75 & 0.66 & 3.24 & 0.47 & 2.27 & 0.49 & 2.27 & 0.48 & 2.20 & 0.49 & 1.93 \\
\hline 3 & 0.60 & 5.84 & 0.35 & 3.41 & 0.32 & 3.20 & 0.33 & 3.27 & 0.32 & 3.17 & 0.31 & 2.73 \\
\hline Sum & 2.96 & 13.72 & 1.89 & 8.9 & 1.30 & 7.11 & 1.34 & 7.16 & 1.33 & 6.97 & 1.33 & 6.22 \\
\hline
\end{tabular}

$$
A\left(\theta_{2}\right)=A_{0}+\theta_{21} A_{1}+\theta_{22} A_{2}+\theta_{23} A_{3}+\theta_{24} A_{4}+\theta_{25} A_{5}+\theta_{26} A_{6},
$$

where

$$
\begin{aligned}
& E_{0}=\operatorname{diag}\left\{1,1,1, m_{1}, m_{2}, m_{3}\right\}, E_{1}=m_{1}[0,0,0,1,0,0]^{T}[0,0,0,1,0,0], \\
& E_{2}=m_{2}[0,0,0,0,1,0]^{T}[0,0,0,0,1,0], E_{3}=m_{3}[0,0,0,0,0,1]^{T}[0,0,0,0,0,1], \\
& B_{\omega 0}=\left[\begin{array}{lllllll}
0 & 0 & 0 & -m_{1}-m_{2} & -m_{3}
\end{array}\right]^{T}, B_{\omega 1}=-m_{1}\left[\begin{array}{llllll}
0 & 0 & 0 & 1 & 0 & 0
\end{array}\right]^{T}[1] \text {, } \\
& B_{\omega 2}=-m_{2}\left[\begin{array}{llllll}
0 & 0 & 0 & 0 & 1 & 0
\end{array}\right]^{T}[1], B_{\omega 3}=-m_{3}\left[\begin{array}{llllll}
0 & 0 & 0 & 0 & 0 & 1
\end{array}\right]^{T}[1] \text {, } \\
& A_{0}=\left[\begin{array}{cccccc}
0 & 0 & 0 & 1 & 0 & 0 \\
0 & 0 & 0 & 0 & 1 & 0 \\
0 & 0 & 0 & 0 & 0 & 1 \\
-k_{1}-k_{2} & k_{2} & 0 & -c_{1}-c_{2} & c_{2} & 0 \\
k_{2} & -k_{2}-k_{3} & k_{3} & c_{2} & -c_{2}-c_{3} & c_{3} \\
0 & k_{3} & -k_{3} & 0 & c_{3} & -c_{3}
\end{array}\right] \\
& A_{1}=k_{1}[0,0,0,-1,0,0]^{T}[1,0,0,0,0,0], A_{2}=k_{2}[0,0,0,-1,1,0]^{T}[1,-1,0,0,0,0] \text {, } \\
& A_{3}=k_{3}[0,0,0,0,-1,1]^{T}[0,1,-1,0,0,0], A_{4}=c_{1}[0,0,0,-1,0,0][0,0,0,1,0,0]^{T}, \\
& A_{5}=c_{2}[0,0,0,-1,1,0][0,0,0,1,-1,0]^{T}, A_{6}=c_{3}[0,0,0,0,-1,1][0,0,0,0,1,-1]^{T} .
\end{aligned}
$$

For subsequent comparison, a robust state feedback controller for the system with uncertainties in the damping and stiffness $\left(\theta_{1 i}=0,\left|\theta_{2 i}\right| \leqslant 0.4\right)$ is designed at first. We solve the LMIs Eq. (24) with a giving $\gamma=1, \beta=0.8$, and obtain the following controller gain matrix based on $K=Z S^{-T}$,

$$
K=10^{5} \times\left[\begin{array}{llllll}
-2.4008 & -0.1070 & 0.4731 & -0.0750 & -0.1283 & -0.1011
\end{array}\right]
$$

For description in brevity, we denote this designed controller as Controller I thereafter.

In order to verify the dynamics of the closed-loop system, the EI Centro earthquake (north-south component, 1940) scaled to a maximum acceleration of $0.112 \mathrm{~g}$, is used as the input excitation. Control performance of the proposed Controller I is compared with other controllers from the viewpoint of maximum responses (maximum interstorey drifts $d_{i}(\mathrm{~cm})$ and maximum absolute accelerations $\ddot{x}_{i}\left(\mathrm{~m} / \mathrm{s}^{2}\right)$ ) in Table 1. it is observed from this table that control performances of saturation controllers such as the MBBC [20], the SSMC [21], the RSC [10], and the proposed Controller I are quite remarkable in comparison with that of the LQR controller, and in the reduction of the interstorey accelerations, the proposed Controller I is the most effective.

Table 2 shows the control performances of the proposed Controller I on maximum response values of the system in comparison with that of the SSMC and RSC in the case of parameter uncertainties with $\theta_{2 i}=0.4(i=1,2, \cdots, 6)$ and $\theta_{2 i}=-0.4(i=1,2, \cdots, 6)$. Obviously, the Controller I proposed in this paper is still the most effective one in the maximum responses reduction. Furthermore, it is worth mentioning that the theorems in this paper are not only effective for LTI systems [10], but also effective for LTV systems.

Now, consider the system without uncertainties and input delay $\tau=25 \mathrm{~ms}$, by solving the LMIs Eq. (7) with a giving $\gamma=5, \beta_{1}=15, \beta_{2}=20$, we can obtain the following controller gain matrix based on $K=Z S^{-T}$ :

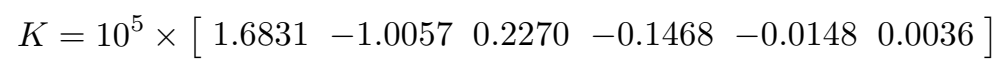


Table 2

Maximum response values for LTI system

\begin{tabular}{|c|c|c|c|c|c|c|c|c|}
\hline \multirow[t]{2}{*}{ Storey } & \multicolumn{4}{|c|}{$\theta_{2 i}=0.4(i=1,2, \cdots, 6)$} & \multicolumn{4}{|c|}{$\theta_{2 i}=-0.4(i=1,2, \cdots, 6)$} \\
\hline & $\begin{array}{c}\text { No control } \\
d_{i}\end{array}$ & $\begin{array}{c}\mathrm{SSMC} \\
d_{i} \\
\end{array}$ & $\begin{array}{c}\mathrm{RSC} \\
d_{i} \\
\end{array}$ & $\begin{array}{c}\text { Controller I } \\
d_{i} \\
\end{array}$ & $\begin{array}{c}\text { No control } \\
d_{i}\end{array}$ & $\begin{array}{c}\mathrm{SSMC} \\
d_{i} \\
\end{array}$ & $\begin{array}{c}\mathrm{RSC} \\
d_{i} \\
\end{array}$ & $\begin{array}{c}\text { Controller I } \\
d_{i} \\
\end{array}$ \\
\hline 1 & 0.70 & 0.31 & 0.31 & 0.30 & 1.64 & 1.05 & 1.06 & 1.05 \\
\hline 2 & 0.57 & 0.28 & 0.28 & 0.28 & 1.34 & 0.97 & 0.97 & 0.81 \\
\hline 3 & 0.34 & 0.20 & 0.19 & 0.19 & 0.79 & 0.61 & 0.61 & 0.51 \\
\hline sum & 1.61 & 0.79 & 0.78 & 0.77 & 3.77 & 2.63 & 2.64 & 2.37 \\
\hline
\end{tabular}

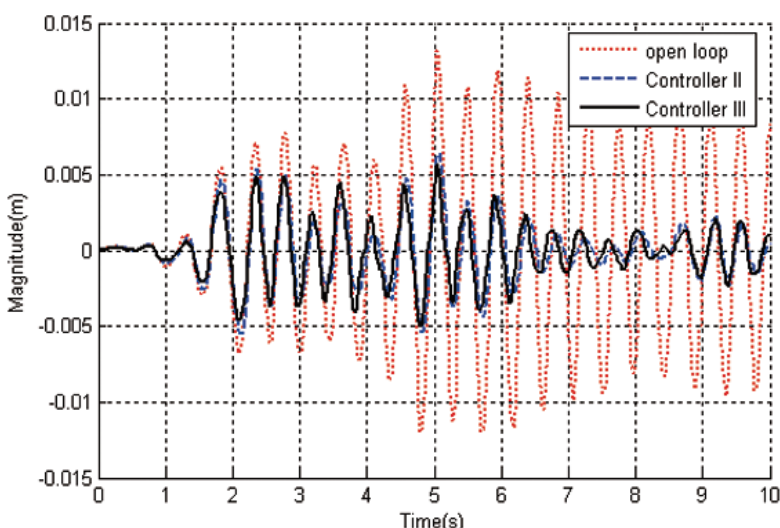

Fig. 2. The displacements of the fist floor $\left(\theta_{1 i}=0, \theta_{2 i}=0, \tau=\right.$ $25 \mathrm{~ms})$.

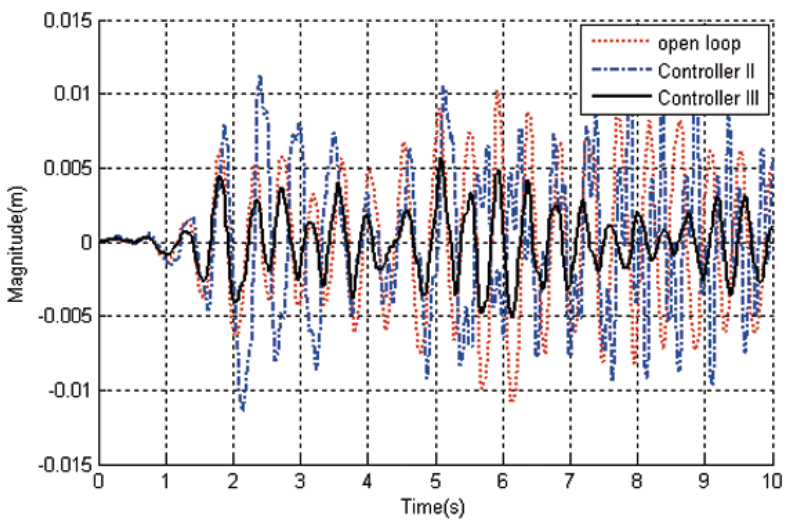

Fig. 4. The displacements of the first floor $\left(\theta_{1 i} \leqslant 0.4, \theta_{2 i} \leqslant 0.2\right.$, $\tau=25 \mathrm{~ms}$ ).

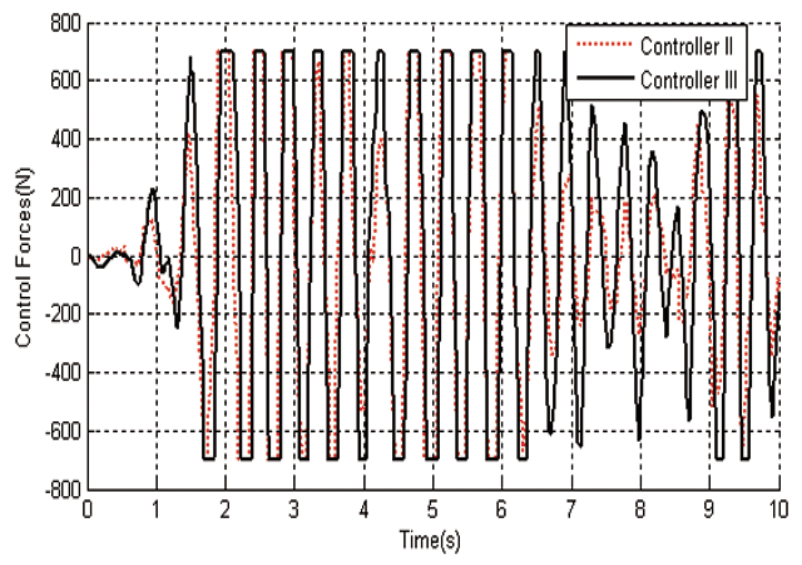

Fig. 3. Comparisons of the control forces of the two controllers.

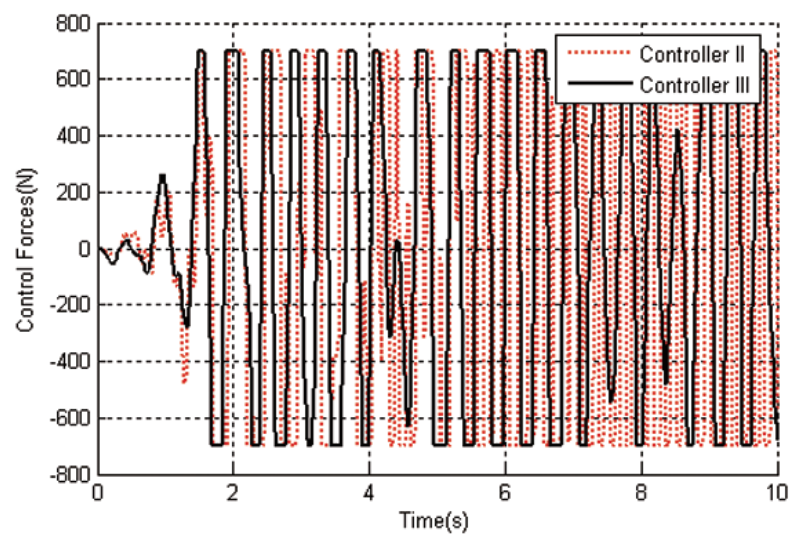

Fig. 5. Comparisons of the control forces of the two controllers.

For description in brevity, we denote this designed controller as Controller II thereafter.

Considering the system with the time-varying uncertainties in the following uncertain intervals: $\left(\left|\theta_{1 i}\right| \leqslant 0.4\right.$, $\left.\left|\theta_{2 i}\right| \leqslant 0.2\right)$. By defining $\gamma=5, \tau=25 \mathrm{~ms}, \beta_{1}=4.9$, and $\beta_{2}=110$, we use Matlab LMI toolbox to solve the LMIs Eq. (17), and obtain the system state feedback controller gain matrix is

$$
K=10^{5} \times\left[\begin{array}{llllll}
3.5527 & -2.5046 & -0.1771 & -0.0170 & -0.0311 S & -0.0472
\end{array}\right]
$$

And we denote this controller as Controller III for brevity.

The earthquake data mentioned above is used as the input excitation. Figure 2 shows the first floor interstorey drift of the three-storey building system, including the open-loop system $(u(t)=0)$ in dotted line, and the closedloop systems equipped with Controllers II and III in dashed and solid lines, respectively. The interstorey drift of 
the other two storeys have the similar varying trend, which are omitted here for brevity. In addition, the velocities output of the three storeys can give us the same information to explain our results, which are also omitted here for brevity. From Fig. 2, we can obtain that the closed-loop responses of the first floor interstorey drift realized by either Controller II or Controller III are much better than the open-loop responses. The control forces of Controllers II and III are depicted in Fig. 3. When the uncertainties mentioned above $\left(\left|\theta_{1 i}\right| \leqslant 0.4,\left|\theta_{2 i}\right| \leqslant 0.2\right)$ are introduced, the first floor interstorey drift responses under the same earthquake are plotted in Fig. 4. It is observed that the closed-loop responses realized by Controller II are worse than the open-loop one, but on the contrary, the closed-loop response realized by Controller III is still better than the open-loop responses no matter the uncertainties introduced. The control forces for this uncertain case are depicted in Fig. 5.

\section{Conclusion}

In this paper, we have addressed the robust active vibration control for a class of earthquake-excited structural systems with time delay and saturation in the control input channel and parameter uncertainties appearing in all the mass, damping and stiffness matrices. Firstly, by using the linear combination of some matrices to describe the system's uncertainties, a new system uncertainties description is developed. Then by employing a linear varying parameter $\alpha(x(t))$, the input saturation model is described as a LPV model. Furthermore, based on parameter-dependent Lyapunov theory and linear matrix inequality technique, the LMIs-based conditions for the closed-loop system to be stable are deduced. By solving those conditions, the controller, considering the actuator saturation, input time delay and parameters uncertainties, is obtained. Finally, a three-storey linear building structure under earthquake excitation is considered and simulation results are given to show the effectiveness of the proposed controllers, furthermore the controller's structure is simple and it is easily realized in the practical engineering.

\section{Acknowledgements}

This work was supported by the Natural Science Foundations (No.51064008; No.51177066) and Jiangxi provincial National Science Foundation (No.GJJ08273) of China.

\section{References}

[1] T.T. Soong and G.P. Cimellaro, Future directions in structural control, Structural Control and Health Monitoring 16(1) (2009), 7-16.

[2] J.F. Sarracini and S.A. Luiz, Reduced model in $H_{\infty}$ vibration control using linear matrix inequalities, Shock and Vibration 13(4-5) (2006), 469-484.

[3] H. Du and N. Zhang, $H_{\infty}$ control for buildings with time delay in control via linear matrix inequalities and genetic algorithms, Engineering Structures 30(1) (2008), 81-92.

[4] M.C. Pai and S. Alok, Asymptotic stability for coupled modal sliding mode control of vibration in a flexible structure, Shock and Vibration 16(3) (2009), 319-324.

[5] A.A. El-Badawy, A.H. Nayfeh and H. VanLandingham, Neural network identification and control of a parametrically excited structural dynamic model of an F-15 tail section, Shock and Vibration 7(6) (2000), 355-361.

[6] M. Chtiba Ouled, S. Choura and S. El-Borgi, Passive control of flexible structures by confinement of vibrations, Shock and Vibration 14(5) (2007), 321-337.

[7] D.V. Balandin and M.M. Kogan, LMI-based optimal attenuation of multi-storey building oscillations under seismic excitations, Structural Control and Health Monitoring 12(2) (2005), 213-224.

[8] Y. Chen, W. Zhang and H. Gao, Finite frequency $H_{\infty}$ control for building under earthquake excitation, Mechatronics 20(1) (2010), $128-142$.

[9] C. Loh, P. Lin and N. Chung, Experimental verification of building control using active bracing system, Earthquake Engineering and Structural Dynamics 28(10) (1999), 1099-1119.

[10] C.W. Lim, Y.J. Park and S.J. Moon, Robust saturation controller for linear time-invariant system with structured real parameter uncertainties, Journal of Sound and Vibration 294(1-2) (2006), 1-14.

[11] C.W. Lim, Active vibration control of the linear structure with an active mass damper applying robust saturation controller, Mechatronics 18 (2008), 391-399.

[12] H. Li, H. Gao and P. Shi. New passivity analysis for neural networks with discrete and distributed delays, IEEE Transactions on Neural Networks 21(11) (2010), 1842-1847. 
[13] H. Du and N. Zhang, Energy-to-peak control of seismic-excited buildings with input delay, Structural Control and Health Monitoring 14(7) (2007), 947-970.

[14] H. Li, H. Liu, H. Gao and P. Shi, Reliable fuzzy control for active suspension systems with actuator delay and fault, IEEE Transactions on Fuzzy Systems 20(2) (2012), 342-357.

[15] D.S. Bernstein and A.N. Michel, A Chronological bibliography on saturating actuators, International Journal of Robust and Nonlinear Control 5(5) (1995), 375-380.

[16] F. Weng and J. Mao, Parameter-dependent vibration-attenuation controller design for electro- hydraulic actuated linear structural systems, Earthquake Engineering and Engineering Vibration 11(1) (2012), 73-82.

[17] X.M. Zhang, H. Ding and S.H. Chen, Interval finite element method for dynamic response of closed-loop system with uncertain parameters, International Journal for Numerical Methods in Engineering 70(5) (2007), 543-562.

[18] H.R. Karimi, M. Zapateiro and N. Luo, An LMI approach to vibration control of base-isolated building structures with delayed measurements, International Journal of Systems Science 41(12) (2010), 1511-1523.

[19] G. Cai, J. Huang, F. Sun and C. Wang, Modified sliding-mode bang-bang control for seismically excited linear structures, Earthquake Engineering and Structural Dynamics 29(11) (2000), 1647-1657.

[20] Z. Wu and T.T. Soong, Modified bang-bang control law for structural control implementation, Journal of Engineering Mechanics-ASCE 122(8) (1996), 771-777.

[21] J.N. Yang, J.C. Wu and A.K. Agrawal, Sliding mode control for seismically excited linear structures, Journal of Engineering MechanicsASCE 121(12) (1995), 1386-1390. 

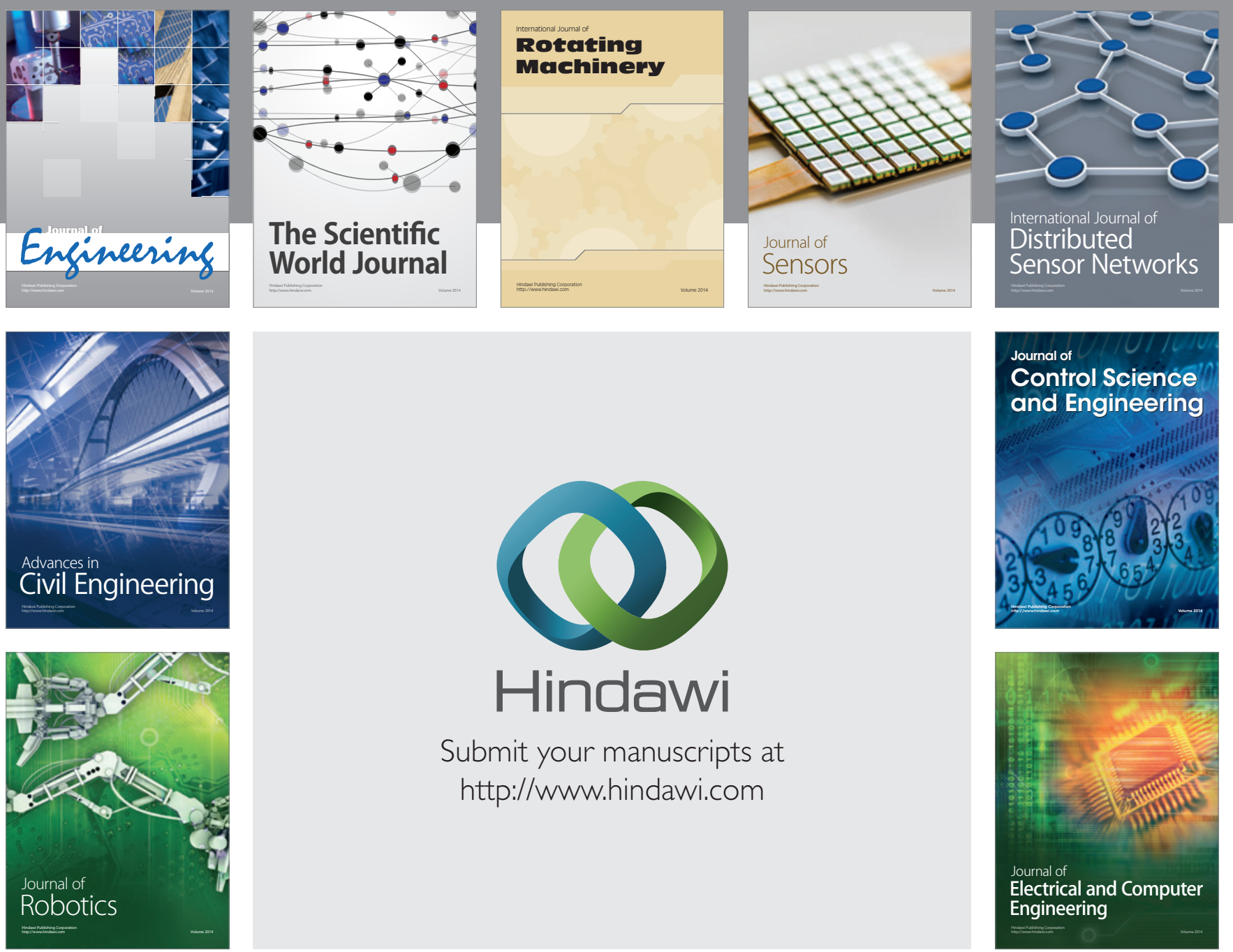

Submit your manuscripts at

http://www.hindawi.com
